\title{
Cholesterol biosynthesis inhibitor RO 48-8071 inhibits viability of aggressive cancer cells
}

\author{
Yayun Liang', Xiaoqin Zou ${ }^{2}$ and Salman M Hyder ${ }^{1 *}$ \\ ${ }^{1}$ Department of Biomedical Sciences and Dalton Cardiovascular Research Center, University of Missouri, Columbia, MO, USA \\ ${ }^{2}$ Dalton Cardiovascular Research Center, Department of Physics and Astronomy, Department of Biochemistry, Institute for Data Science and Informatics, University \\ of Missouri, Columbia, MO 65211, USA
}

\begin{abstract}
Background: RO-48-8071 (4-Bromophenyl)[2-fluoro-4-[[6-(methyl-2-propen-1-ylamino)hexyl]oxy]phenyl]methanone) (RO) is a cholesterol lowering drug that targets oxidosqualene cyclase in the cholesterol biosynthesis pathway. We recently reported anti-cancer properties for RO in breast and prostate cancer cells.

Methods: The present study describes that RO 48-8071 is also an effective agent against aggressive drug-resistant colon, pancreas and lung cancer cells. We examined whether RO could inhibit the growth of cancer cells in vitro using a well-established cell viability assay in a short time frame (24-48 h).

Results: Treatment of 7 different aggressive cancer cell lines with RO for 48 h significantly reduced their viability (IC50 range 3.3 to $13.68 \mu \mathrm{M}$ ).

Conclusion: As reported earlier, a long term-assay (5-7 days) showed that nM levels of RO also effectively reduced the viability of breast and prostate cancer cells, though this remains to be investigated for the cell lines reported here. RO warrants further investigation as an effective anti-cancer compound.
\end{abstract}

\section{Introduction}

We recently showed that the cholesterol biosynthesis inhibitor RO 48-8071 is a very effective therapeutic agent against breast and prostate cancer cells, both in vitro and in vivo [1,2]. RO 48-8071 inhibits oxidosqualene cyclase, an enzyme downstream of HMGCoA reductase; latter is the rate limiting enzyme in the cholesterol biosynthetic pathway. RO 48-8071 was also effective at low doses (nM range) over 5-7 days and did not influence normal cells [1,2]. Others have shown that RO 48-8071 is also an effective PI3-Kinase inhibitor in different types of cancer, as well as being an antiangiogenic agent that restricts the growth of endothelial cells [3]. Thus RO 48-8071 appears to be a multifunctional anti-cancer agent that blocks the growth of both epithelial and endothelial cells. In this communication we would like to report that RO 48-8071 is also effective in vitro against other lethal types of drug-resistant cancer, including lung, colon and pancreatic cancer. These cancer types are difficult to treat and often metastasize, leading to patient death.

\section{Materials and methods}

\section{Cell lines and culture}

All cell-culture studies were approved by the University of Missouri Institutional Environmental Health and Safety Board (Columbia, MO, USA). All cell lines were obtained from the American Type Culture Collection (Manassas, VA, USA). Cells were grown as described in legends in DME/F12 medium or RPMI-1640, Iscove's Modified Dulbecco's Medium (IMDM), or F-12K medium supplemented with $10 \%$ or $20 \%$ fetal bovine serum (FBS; Sigma-Aldrich, St. Louis, MO, USA), at $37^{\circ} \mathrm{C}$ in a humidified atmosphere of $5 \% \mathrm{CO} 2$ and harvested for different experiments with $0.05 \%$ trypsin-EDTA (ThermoFisher Scientific, Waltham, MA, USA). Cells were washed with phosphate- buffered saline (PBS) or FBS-free medium before being treated in fresh medium containing $5 \%$ or $10 \%$ FBS described in each figure.

\section{Cell viability assay}

We utilized the well-established SRB cell viability assay $[4,5]$ to determine the inhibitory effect of $\mathrm{RO} 48-8071$ on drug-resistant colon, lung and pancreatic cells of aggressive nature [6-12]. The SRB assay quantitates protein content of surviving cells as an index of cell growth and viability, as described in our previous publications [1,2,13]. Six wells were used for each concentration, and experiments were performed three to five times in order to calculate IC50 values. Representative experiment is shown in Figures 1-3 and IC50 values are listed in Table 1. Viability of 7 different cancer cell lines was inhibited by RO 48-8071.

\section{Statistical analysis}

Differences among groups were tested using one-way analysis of variance (ANOVA). SigmaPlot software (version 14) was used for statistical analysis. Data are reported as mean \pm standard error of the mean (SEM). For all comparisons, $\mathrm{P}<0.05$ was considered significant. The assumption of the ANOVA was examined, and if necessary, a nonparametric measure based on ranks was used. If normality failed, Kruskal-Wallis one-way ANOVA on ranks was used in place of regular ANOVA. In cases where a significant effect was shown by ANOVA

${ }^{\star}$ Correspondence to: Salman M Hyder, Dalton Cardiovascular Research Center University of Missouri-Columbia, 134 Research Park Drive, Columbia, MO 65211, USA, E-mail: hyders@missouri.edu

Key words: colon cancer, pancreatic cancer, lung cancer, cell viability

Received: September 05, 2020; Accepted: September 15, 2020; Published: September 22, 2020 


\section{Colon Cancer}
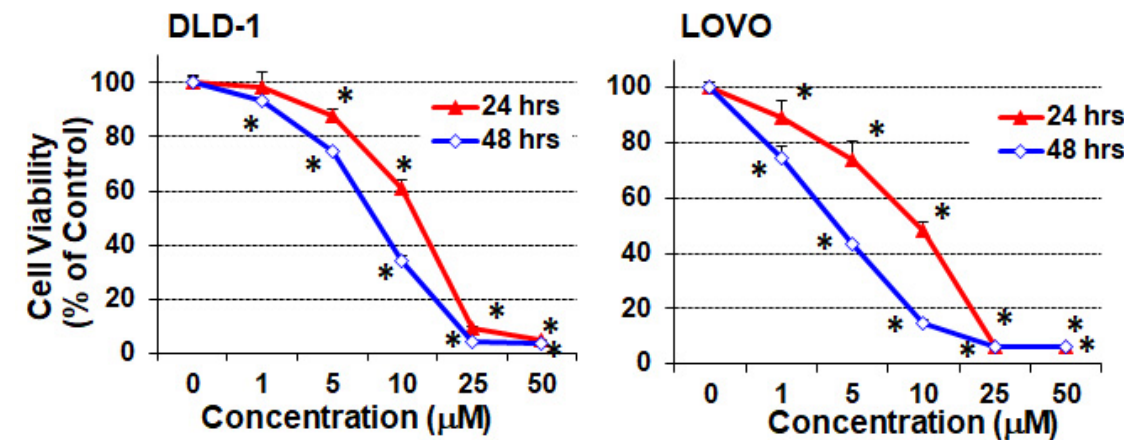

Figure 1. Effect of RO 48-8071 on colon cancer cell line viability. (A) DLD-1 cells $\left(4.0 \times 10^{3} /\right.$ well) and (B) LoVo cells $\left(6.0 \times 10^{3} /\right.$ well) were seeded into a $96-$ well plate overnight. Cells were washed with culture medium devoid of FBS, then treated for 24 or 48 hours with RO $48-8071$. For DLD- 1 cells, seeding was in RPMI- $1640+10 \%$ FBS and treatment was in RPMI- $1640+5 \%$ FBS. For LeVo cells, the seeding medium was $10 \%$ FBS F-12K, and treatment was in 5\% FBS F12K. Cell viability was determined by SRB assays. Data are shown as the mean \pm SEM $(\mathrm{n}=6)$. *Significantly different from control group; $P<0.05$ (ANOVA)

\section{Lung Cancer}
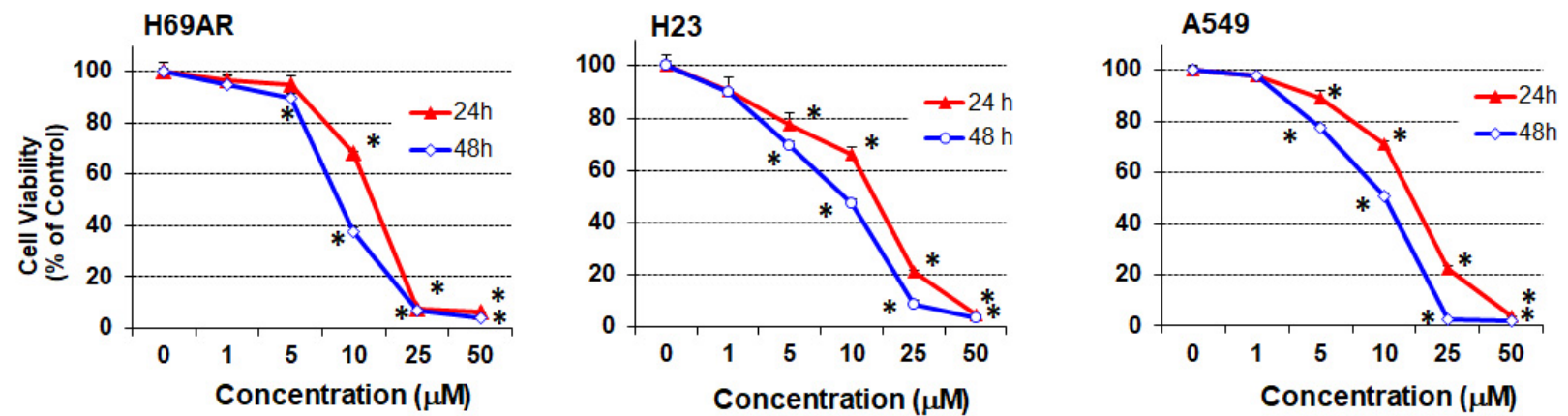

Figure 2. Effect of RO 48-8071 on lung cancer cell line: (A) H69AR cells $\left(1 \times 10^{4} /\right.$ well $)$ and (B) NCI-H23 cells (3.5 $\times 10^{3} /$ well), (C) A549 cells (3.0 $\times 10^{3} /$ well) were seeded into a $96-$ well plate overnight. Cells were washed with culture medium devoid of FBS, then treated for 24 or 48 hours with RO $48-8071$. For H69AR cells, the seeding medium was $20 \%$ FBS RPMI- 1640 , and $10 \%$ FBS RPMI-1640 for treatment. For NCI-H23 cells, the seeding medium was 10\% FBS RPMI-1640, and 5\% FBS RPMI-1640 for treatment. For A549 cells, the seeding medium was $10 \%$ FBS F-12K, and 5\% FBS F-12K for treatment. Cell viability was determined by SRB assay. Data are shown as the mean \pm SEM ( $=6$ ). *Significantly different from control group; $P<0.05$ (ANOVA)

\section{Pancreatic Cancer}
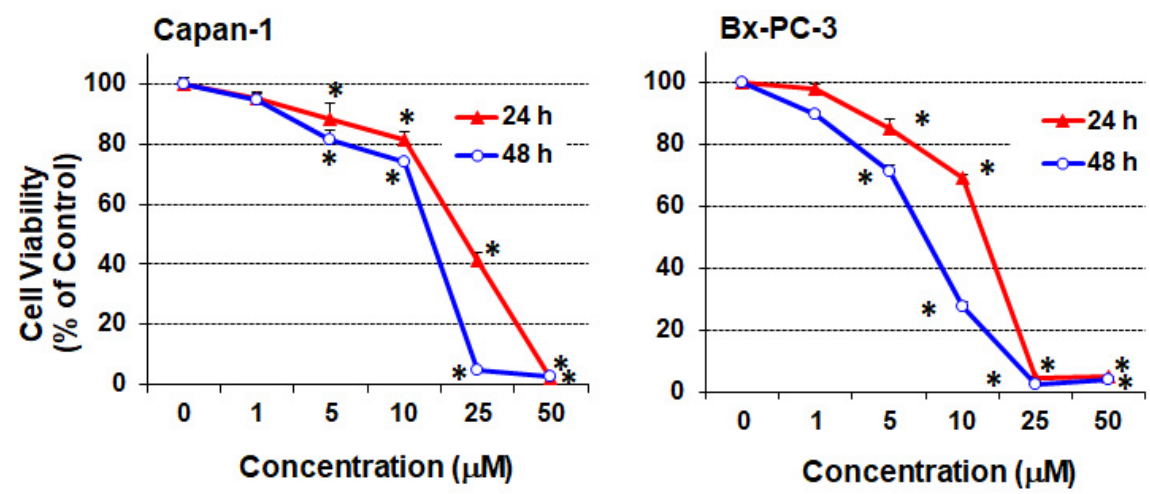

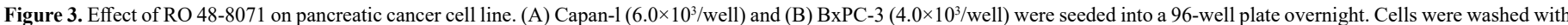
culture medium devoid of FBS, then treated for 24 or 48 hours with RO 48-8071. For Capan-1 cells, the seeding medium was IMDM with $20 \%$ FBS, and IMDM with $10 \%$ FBS IMDM for treatment. For BxPC-3 cells, the seeding medium was RPMI-I640+10\% FBS, and RPMI $+5 \%$ FBS for treatment. Cell viability was determined by SRB assay. Data are shown as the mean \pm SEM ( $\mathrm{n}=6) *$ Significantly different from control group; $P<0.05$ (ANOVA) 
Table 1. IC50 $(\mu \mathrm{M})$ of RO 48-8071 in cancer cell lines

\begin{tabular}{|c|c|c|c|}
\hline Cancer type & Cell lines & $\mathbf{2 4}$ hours & $\mathbf{4 8}$ hours \\
\hline Colon & DLD-1 & $11.49 \pm 0.24$ & $6.93 \pm 0.41$ \\
\hline & LOVO & $9.46 \pm 0.07$ & $3.30 \pm 0.54$ \\
\hline Lung & H69AR & $10.84 \pm 0.61$ & $8.11 \pm 0.36$ \\
\hline & H23 & $14.19 \pm 1.28$ & $10.28 \pm 0.30$ \\
\hline & A549 & $13.51 \pm 0.93$ & $9.26 \pm 0.45$ \\
\hline Pancreas & Capan-1 & $18.55 \pm 1.62$ & $13.68 \pm 0.17$ \\
\hline & BxPC-3 & $11.38 \pm 0.62$ & $7.11 \pm 0.10$ \\
\hline
\end{tabular}

(F-ratio, $\mathrm{P}<0.05$ ), the Student-Newman-Keuls multiple comparison test was employed to compare the means of the individual groups.

\section{Results}

Figure 1 shows the effect of RO 48-8071 on colon cancer cell lines. The DLD- 1 cell line, derived from a male patient, is of the colorectal adenocarcinoma type and contains a mutation in the p53 gene. The LOVO cell line is also of the colorectal adenocarcinoma type and is derived from a metastatic lesion.

Figure 2 shows growth inhibitory properties of RO 48-8071 on 3 different lung cancer cell lines. H69AR is a drug-resistant cell line derived from small cell lung cancer. H23 is also an adenocarcinoma cell line with a mutation in the p53 gene. A549 is derived from a lung carcinoma of a patient.

Figure 3 shows the effect of RO 48-8071 in suppressing pancreatic cancer cell viability. Capan-1 cell line is derived from a metastatic site (liver) of a pancreatic cancer patient. BxPC-3 cell line is derived from a pancreatic adenocarcinoma.

Table 1 shows the IC50 values for RO 48-8071 against colon, lung and pancreatic cell lines drawn from experiments shown in Figures 1-3. While all cell lines were sensitive to RO 48-8071 the colon cancer cell lines showed great sensitivity to the drug.

\section{Discussion}

Since many cancers become resistant to current chemotherapeutic approaches, there is a need to discover new pathways and ligands that could be used as alternatives to cure the disease. With this in mind we identified a cholesterol biosynthesis inhibitor, RO 48-8071, as a potent inhibitor of both breast and prostate cancer cells [1,2], as well as ovarian cancer cells (data to be presented elsewhere). RO 48-8071 induced apoptosis of cancer cells and also activated estrogen receptor-beta, which is known to have anti-proliferative properties in many cell types $[1,2]$. Since low $(\mathrm{nM})$ concentrations of RO blocked tumor cell growth over a 5-7-day study, toxicity of the compound would likely be very low. This was borne out in in vivo studies [1,2] and further supported by our observations that RO 48-8071 had no effect on normal cell lines [1,2]. It was our aim in our initial studies to ascertain whether RO 48-8071 also reduces the growth properties of cancer cell lines derived from lethal types of cancer, especially drug resistant cancer cells. To this end we utilized an assay that permitted us to determine the effects of RO 488071 on tumor cell viability. Based on our findings we predict that RO 48-8071 will also be highly effective in vivo, though its in vivo effects were not examined in the present study. Our aim here is to inform researchers of the potential of RO 48-8071 and to highlight an extremely promising compound whose effects warrant further investigation in the form of human clinical trials.
Our observations show that RO 48-8071 effectively disrupts the progression of colon, lung and pancreatic cancer cells that grow aggressively and for which there are few treatment options. Colon cancer cells appear to be most sensitive to RO 48-8071 suggesting that perhaps this drug enters these cells most effectively although this remains to be proven. In future studies the mechanism of action of RO 48-8071 will be determined in the cell lines tested. We predict that RO 48-8071 will induce apoptosis as well as inhibit PI3-kinase activity as previously described [1-3]. Future in vivo therapeutic studies are also important to establish with the cell lines described.

\section{Conclusion}

We previously published our findings that RO 48-8071 inhibits breast and prostate cancer cells. Based on our current studies we contend that RO 48-8071 is an effective inhibitor of multi-cancer cells, including those that are drug-resistant, and that its anti-cancer properties warrant further investigation, perhaps in the form of human clinical trials.

\section{Acknowledgments}

Financial support for research was provided by a peer-reviewed faculty research grant from the College of Veterinary Medicine, University of Missouri. SMH is the Zalk Missouri Professor of Tumor Angiogenesis.

\section{Disclosure}

The authors and the University of Missouri, Columbia hold a Patent on anti-cancer properties of RO 48-8071.

\section{References}

1. Liang Y, Mafuvadze B, Aebi JD, Hyder SM (2016) Cholesterol biosynthesis inhibitor RO 48-8071 suppresses growth of hormone- dependent and castration-resistant prostate cancer cells. Onco Targets and Therapy 9: 3223-3232

2. Liang Y, Besch-Williford C, Aebi JD, Mafuvadze B, Cook MT, et al. (2014) Cholestero biosynthesis inhibitors as potent novel anti-cancer agents: suppression of hormonedependent breast cancer by the oxidosqualene cyclase inhibitor RO 48-8071. Breast Cancer Res Treat 146: 51-62.

3. Oliaro-Bosso S, Meda C, Di Nicolantonio F, Bussolino F, Balliano G, et al. (2015) The cholesterol biosynthesis enzyme oxidosqualene cyclase is a new target to impair tumour angiogenesis and metastasis dissemination. Sci Rep 5: 9054. [Crossref]

4. Rubinstein LV, Shoemaker RH, Paull KD, Simon RM, Tosini S, et al. (1990) Comparison of in vitro anticancer-drug-screening data generated with a tetrazolium assay versus a protein assay against a diverse panel of human tumor cell lines. $J$ Natl Cancer Inst. 82: 1113-1118.

5. Skehan P, Storeng R, Scudiero D, Monks A, McMahon J, et al. (1990) New colorimetric cytotoxicity assay for anti-cancer-drug screening. J Natl Cancer Inst. 82: 1107-1112.

6. Capan-1: Human pancreatic adenocarcinoma cell line (ATCC HTB-79) the Capan-1 cells are resistant to 5 -fluorouracil and contains an inactivating mutant p53.

7. Simon B, Weine R, Hohne M, Watz J, Schmidt J, et al. (1994) Frequent alterations of the tumor suppressor genes p53 and DCC in human pancreatic carcinoma. Gastroenterology 106: 1645-1651.

8. Gao Y, Dorn P, Liu S, Deng H, Hall SRR, et al. (2019) Cisplatin-resistant A549 non-small cell lung cancer cells can be identified by increased mitochondrial mass and sensitive to pemetrexed treatment. Cancer Cell International 19: 317-331. [Crossref]

9. Toffoli G, Corona G, Gigante M, Boiocchi M (1996) Inhibition of Pgp activity and cell cycle-dependent chemosensitivity to doxorubicin in the multidrug-resistant LoVo human colon cancer cell line. European Journal of Cancer 32: 1591-1597.

10. Caster JM, Sethi M, Kowalczyk SW, Xi ET, Hyder SN (2015) Nanoparticle delivery of chemosensitizers improve chemotherapy efficacy without incurring additional toxicity. Nanoscale 7: 2805-2811.

11. Nath S, Daneshvar K, Roy LD, Grover P, Kidiyoor A, et al. (2013) MUC1 induces drug resistance in pancreatic cancer cells via upregulation of multidrug resistance genes. Oncogenesis 2: 51-59. [Crossref] 
Liang Y (2020) Cholesterol biosynthesis inhibitor RO 48-8071 inhibits viability of aggressive cancer cells

12. Kramer R, Weber TK, Morse B, Arceci R, Staniunas R, et al. (1993) Constitutive expression of multidrug resistance in human colorectal tumours and cell lines. $\mathrm{BrJ}$ Cancer 67: 959-968.
13. Grinter SZ, Liang Y, Huang SY, Hyder SM, Zou X (2011) An inverse docking approach for identifying new potential anti-cancer targets. J Molecular Graphics and Modelling 29: 795-799.

Copyright: (C2020 Liang Y. This is an open-access article distributed under the terms of the Creative Commons Attribution License, which permits unrestricted use, distribution, and reproduction in any medium, provided the original author and source are credited. 